\title{
Validation of model-based correction for non-Stokesian tracers
}

\author{
Joshua N. Galler ${ }^{1 *}$, David E. Rival ${ }^{1}$ \\ ${ }^{1}$ Queen's University, Department of Mechanical and Materials Engineering, Kingston, Canada \\ *j.galler@queensu.ca
}

\begin{abstract}

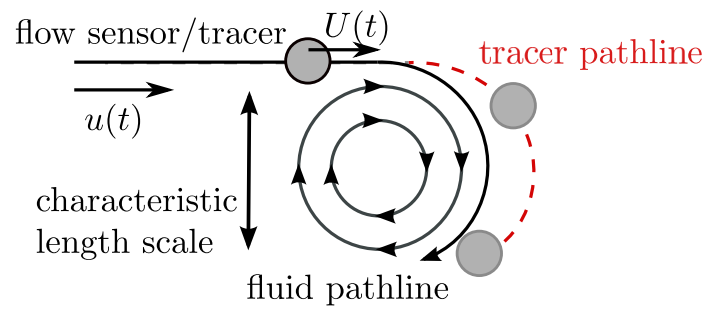

In-situ flow-tracking measurements at scales on the order of $10 \mathrm{~m}^{3}$ and larger remain a challenge. The large size of the tracers required for optical visibility results in an inertial lag and inherently low seeding density. For instance, natural snowfall, fake snow and soap bubbles on the order of $2 \mathrm{~cm}$ have been used as tracers for field measurements and extracted statistical quantities (Nemes et al., 2017; Wei et al., 2021; Rosi et al. 2014). There is also growing interest in networks of sensors for remote-measurement where optical access is impossible (Bolt et al. 2020; Villa et al. 2016). Onboard inertial measurement units (IMU) are a promising tool for high-resolution measurements over large spatial domains without optical access. However, due to the intrinsic lag, a dynamic-model-based correction is required for the tracking of transient phenomena, sketched in figure 1. In the present study, the tracer-velocity correction is evaluated by quantifying the residual error in measured flow velocity after the method of Galler et al. (2021) is applied.

(a) non-ideal tracer response

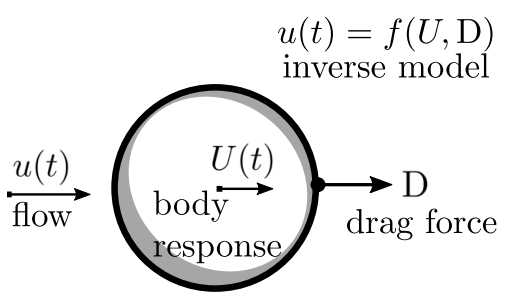

(b) inverse-model approach

Figure 1: a) Sketch of a scenario in which sensor lag hinders the measurement of a coherent structure. Error in local velocity and in fluid trajectories are a result of tracer inertia. b) Sketch of the dynamic system in the laboratory-fixed frame of reference. The applied flow, $u(t)$, causes aerodynamic drag, $D$, and results in a body response, $U(t)$. With a robust model for $D, u$ can be calculated using measured body velocity, $U$.

To apply the dynamic-model-based approach to large tracers, the sensor response was captured in a gust flow. A spherical housing, pictured in figure 2(a), is used to carry the custom IMU and simplify physical modelling. The sphere has a diameter of $10 \mathrm{~cm}$ and the system has a total mass of $12.3 \mathrm{~g}$. Experiments were performed in an open-jet gust wind tunnel with vertical configuration, seen in figure 2(b). The jet outlet has a cross-sectional area of $144 \mathrm{~cm}^{2}$. Air was accelerated from rest to a terminal jet velocity of $u_{f}=15 \mathrm{~m} / \mathrm{s}$. The sensor was optically tracked using a Photron SA-4 high-speed camera and a Python-based computer vision algorithm.

Figure $3(\mathrm{a})$ shows the agreement between IMU and motion-tracking camera, validating the processing algorithm. Figure 3(b)] shows the agreement between the model correction and measured disturbance. Despite the weak response of the body, in significant part due to its drag-to-weight ratio, the flow perturbation was extracted through dynamic modelling. The maximum residual velocity (error) was approximately $15 \%$, a vast improvement over $95 \%$ error observed with the raw sensor output. In addition, while the sensor lagged behind the disturbance, never fully matching the prescribed velocity, the correction reduced the time-lag to approximately $30 \%$. Since the model correction relies on the sensor acceleration as input, the extracted time-scale is more sensitive to the magnitude of the aerodynamic response than the extracted velocity.

In the present study, the feasibility of IMU-based flow measurement is demonstrated using a nonStokesian sensor coupled with a dynamic-model-based correction. The working principle and proof-of- 


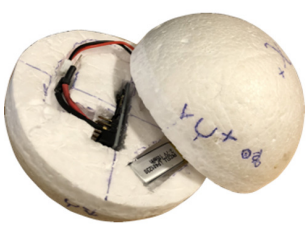

assembled prototype

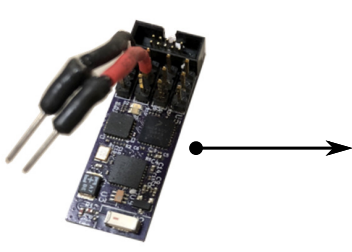

on-board sensor communicates data to base station

(a) IMU flow tracer system
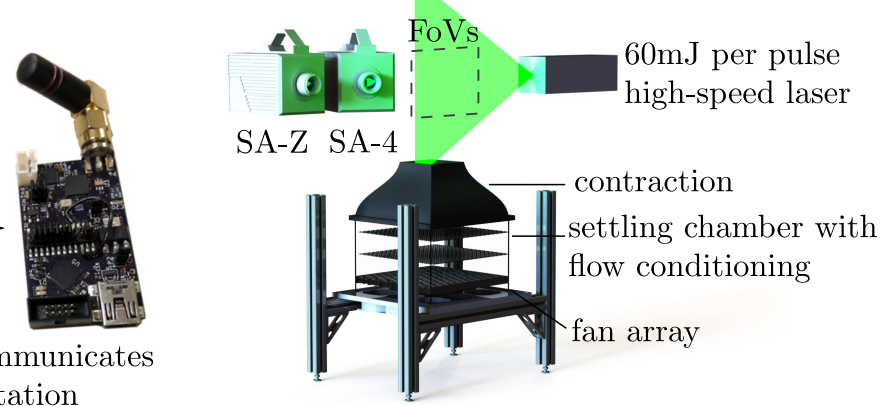

(b) wind tunnel setup

Figure 2: a) Spherical sensor housing, IMU and base station units. b) Vertical open-jet gust wind tunnel facility showing the location of the sphere sensor, high-speed cameras, laser sheet and fields of view (FoVs).
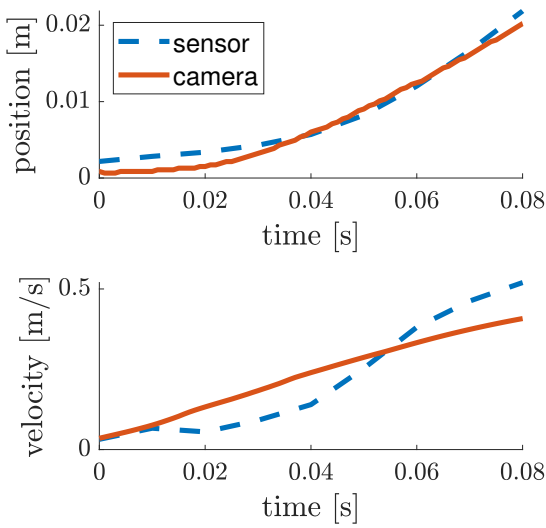

(a) IMU validation

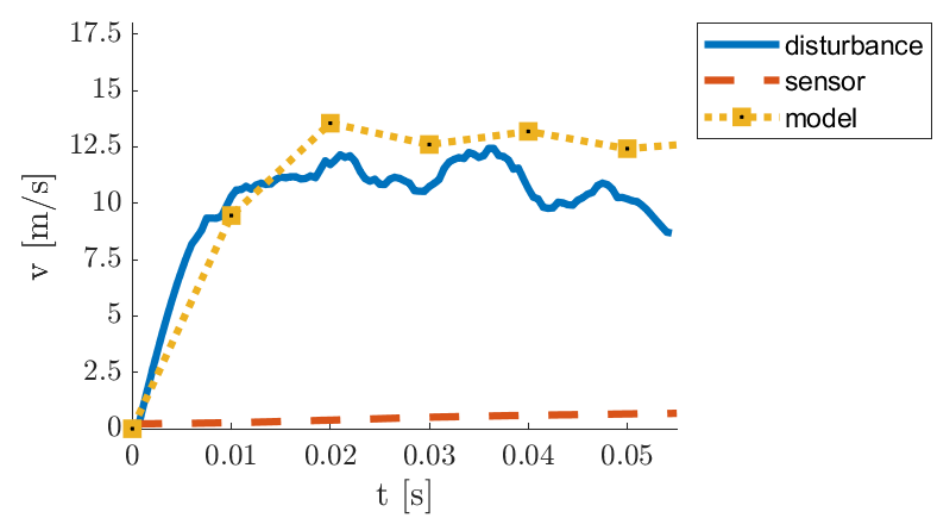

(b) inverse model validation

Figure 3: (a) Position and velocity time histories for the sensor and camera measurements. Good agreement validates the sensor output. (b) Modelled sensor correction against PIV flow velocity measurements. Good agreement suggests the model approach allows for the use of non-Stokesian flow tracer and sensor systems.

concept is presented through in-lab measurements of a rapid gust as a canonical flow perturbation example. Minimum resolvable time scales are shown to be a function of the sensor aerodynamic response.

\section{References}

Bolt M, Prather JC, Horton T, Adams M et al. (2020) Massively deployable, low-cost airborne sensor motes for atmospheric characterization. Wireless Sensor Network 12:1

Galler JN, Weymouth GD, and Rival DE (2021) On the concept of energized mass: A robust framework for low-order force modeling in flow past accelerating bodies. Physics of Fluids 33:057103

Nemes A, Dasari T, Hong J, Guala M, and Coletti F (2017) Snowflakes in the atmospheric surface layer: observation of particle-turbulence dynamics. Journal of Fluid Mechanics 814:592-613

Rosi GA, Sherry M, Kinzel M, and Rival DE (2014) Characterizing the lower log region of the atmospheric surface layer via large-scale particle tracking velocimetry. Experiments in Fluids 55:1736

Villa TF, Gonzalez F, Miljievic B, Ristovski ZD, and Morawska L (2016) An overview of small unmanned aerial vehicles for air quality measurements: Present applications and future prospectives. Sensors $16: 1072$

Wei NJ, Brownstein ID, Cardona JL, Howland MF, and Dabiri JO (2021) Near-wake structure of full-scale vertical-axis wind turbines. Journal of Fluid Mechanics 